\title{
Marcas de la Pandemia: El Derecho a la Educación Afectado
}

\section{The Pandemic Traces: The Stricken Right to Education}

\author{
Guillermo Ramón Ruiz * \\ Universidad de Buenos Aires, Argentina
}

\begin{abstract}
En este trabajo se analizan algunas de las implicancias que ha tenido la pandemia del Covid-19 sobre el ejercicio del derecho a la educación. Se realiza un encuadre que considera el contexto global a través de algunos indicadores recientes sobre los efectos que ha tenido la pandemia en la escolarización, y a partir de ello se intenta elucidar algunas consecuencias sobre el derecho a la educación en función de su contenido en términos pedagógicos, o sea, en la formación y el aprendizaje. En primer lugar, se define el contenido del derecho a la educación desde el enfoque de los derechos humanos y las obligaciones estatales en la materia. En segundo lugar, se realiza un doble análisis del impacto de la pandemia sobre la escolarización masiva, tanto desde una perspectiva macro (al considerar los datos globales) como también desde una perspectiva micro (al contemplar los efectos en los sistemas y en las instituciones de todos los niveles educativos). Se problematizan algunas de las decisiones tomadas por los gobiernos, referidas a la celeridad con que se implementaron programas educación a distancia a través de plataformas digitales, y que han afectado la formación de las personas. Finalmente, se plantean algunos de los escenarios generados y los desafíos que en ellos se evidencian para garantizar el ejercicio de este derecho humano fundamental.
\end{abstract}

Descriptores: Derecho a la educación; Pandemia; Cierre de escuelas; Políticas educativas; Educación a distancia.

This article focusses on the implications that the Coronavirus Pandemic (COVID19) has had on the right to education. From an international point of view, it is taking some educational indicators to see how schooling was affected during the coronavirus outbreaks, and how pedagogical dimensions of the right to education are neglected consequently. At the beginning, I undertook a systematic review of the definition of the concept of right to education from both, the human rights based approach, and also, according to the State responsibilities to guarantee to people the access to formal education. Then, I analyzed the educational consequences of school closures from macro- and micro-level perspectives. The purpose here is to measure the pedagogical implications that distance learning programs (through digital learning platforms) could have on the right to education when they are implemented by governments and institutions, in short time. That is to say without enough teaching planning beforehand. Finally, I explored some scenarios and challenges that States and authorities should face in order to guarantee the right to education as a constitutional human right.

Keywords: Right to education; Pandemic; School closures; Education policies; Distance learning.

*Contacto: gruiz@derecho.uba.ar

ISSN: 2254-3139

www.rinace.net/riejs/

revistas.uam.es/riejs
Recibido: $\quad 2$ de abril 2020

$1^{\text {a }}$ Evaluación: 13 de mayo 2020

$2^{\text {a }}$ Evaluación: 2 de junio 2020

Aceptado: $\quad 10$ de junio 2020 


\section{Encuadre}

La institucionalización de la educación en sistemas escolares de alcance masivo es una forma particular de educación organizada a la luz de la constitución de los Estados nacionales modernos que, a partir de principios del siglo XIX, se ha extendido desde el contexto europeo al americano y que ha alcanzado, durante el siglo $\mathrm{XX}$, una mundialización como forma de instruir a la población infantil y joven de manera compulsiva, tal como ha sido definida por algunas líneas de investigación de la educación comparada (Altbach y Kelly, 1986; Meyer y Ramírez, 2010). El propósito original era la conformación de la ciudadanía nacional, con la peculiaridad de que los diferentes Estados nacionales dieron lugar a diversos tipos de ciudadanía ideal, a través de los desarrollos de sistemas escolares y currículos nacionales. Durante el siglo XX, la complejidad de la vida social y la evolución de los mecanismos de inclusión, cierre y segmentación han tornado más sofisticada la escolarización como institucionalización de la educación. Los procesos de transferencia internacional de discursos y políticas educativas entre diferentes contextos nacionales han contado con auspicios diversos, locales y foráneos, que han ejercido influencias sobre los sistemas nacionales y han generado conflictos entre las comunidades locales como consecuencia del carácter cultural diferencial de las presiones internacionales (Tröhler y Lenz, 2015).

La educación institucionalizada en sistemas escolares, denominada educación formal, constituye así una de las características fundamentales de las sociedades contemporáneas. Ello radica en buena medida en su alcance masivo y en la carga de significados culturales que le confieren legitimidad para definir mitos racionalizados sobre la sociedad y las personas (Meyer y Ramírez, 2010). La organización de los sistemas escolares sobre la base de una estructura académica constituye a su vez uno de los rasgos distintivos de la escolarización moderna y de la distribución de los saberes oficialmente válidos que, definidos por la autoridad estatal, son difundidos entre la población de forma tal que se articulen lazos sociales disímiles entre los diferentes grupos y actores (Ruiz, 2020).

Esa distribución del saber a la vez conforma el núcleo formativo de uno de los derechos humanos fundamentales. Efectivamente, durante el proceso histórico de conformación de los sistemas escolares modernos, el derecho a la educación aparece como uno de los elementos centrales tanto en las definiciones políticas como en los procesos de transferencias internacionales de discursos y prácticas escolares. Primero lo hizo como proclama igualadora de la población, y luego como concepto de las ciencias jurídicas que refiere a uno de los derechos humanos fundamentales. Durante la segunda parte del siglo $\mathrm{XX}$, el derecho a la educación ha sido incluido con centralidad incomparable en la agenda pública, y en los programas de gobiernos de todas las orientaciones ideológicas. Asimismo, concitó acuerdos internacionales que se tradujeron en acciones de alcance global y en compromisos por parte de los Estados, que asumieron las correspondientes obligaciones para garantizarlo, favorecerlo y promoverlo. Se lo considera un derecho social, un derecho humano, un derecho fundamental. Se afirma que su promoción constituye una garantía de desarrollo humano y social, ya que posibilita generar mejores condiciones de empleabilidad de la población y provee al sector productivo de recursos humanos a la par que favorece el desarrollo científico y tecnológico en el nivel superior. La educación como derecho ocupa actualmente un rol destacado en su reconocimiento internacional como parte de los derechos humanos y también en el marco de los Estados. 
En este trabajo se analizan algunos de los efectos que sobre el ejercicio del derecho a la educación han tenido las decisiones que se han tomado para hacer frente a la pandemia del coronavirus disease 2019 (Covid-19), principalmente aquellas que involucran a la instrumentación generalizada de programas de educación a distancia. Se realiza un doble análisis del impacto de la pandemia sobre la escolarización masiva, tanto desde una perspectiva macro (al considerar los datos globales) como también desde una micro (al contemplar los efectos pedagógicos de convertir las clases presenciales en virtuales). Finalmente, se plantean algunos de los escenarios generados y los desafíos que en ellos se evidencian para garantizar el ejercicio de este derecho humano fundamental.

\section{La educación desde el enfoque de derechos humanos y sus implicancias formativas}

Un punto de demarcación -desde el punto de vista histórico- en lo que atañe a la configuración del derecho a la educación estuvo dado por la conformación del derecho internacional de los derechos humanos luego de la finalización de la Segunda Guerra Mundial. En dicho contexto de posguerra la educación pasó a ser considerada uno de los derechos humanos fundamentales de contenido prestacional y, por ende, exige la intervención positiva del Estado para garantizar su goce a todas las personas. La aprobación de la Declaración Universal de los Derechos Humanos (1948), el afianzamiento del derecho internacional sobre derechos humanos, y la consiguiente formulación de los instrumentos internacionales de derechos humanos y su incorporación a los textos constitucionales han posibilitado que nuevos procesos y fenómenos jurídicos ampliasen el debate sobre la naturaleza y los alcances del contenido de este derecho y los correlativos niveles de obligaciones a cargo del Estado.

El derecho a la educación hace referencia no sólo a un derecho de las personas a recibir educación sino, como todo derecho humano, también implica obligaciones estatales específicas (Tomasevski, 2001). Este derecho comprende -desde el plexo normativo internacional- un catálogo de obligaciones a cargo de los Estados nacionales para: respetarlo, protegerlo, cumplirlo, realizarlo y garantizarlo. Para ello debe favorecer un desarrollo normativo para la educación, que contemple tanto el dictado de reglas y procedimientos, así como la también eficaces sistemas administrativos y judiciales, con acceso igualitario (Ruiz, 2020). Podría sostenerse que en la actualidad sigue vigente el planteo histórico iniciado en aquel contexto posterior a la Segunda Guerra Mundial. Su vigencia se evidencia en términos discursivos, pero también a través de las divergentes respuestas que se dan en torno al criterio a adoptar para la distribución social de los recursos que los Estados definen para promover la escolarización masiva de la población infantil, joven y adulta. Más allá de toda discusión sobre las definiciones conceptuales y materiales del contenido del derecho a la educación, es evidente que los sistemas nacionales de escolarización constituyen el indicador más notorio y valorado de este derecho humando y que la expansión, acceso y alcances de la educación formal (en cuanto a retención y graduación de estudiantes) conforman proclamas igualadoras altamente consensuadas entre los países y las personas, por encima de las diferencias ideológicas y culturales.

Ahora bien, en la tarea de conceptualizar el derecho a la educación, se podría tomar como punto de partida el hecho de que todas las personas tienen derecho a la educación debido 
a que las capacidades para interpretar el mundo y actuar en él resultan esenciales para la vida humana. Al considerar a todas las personas, se subraya en este punto el principio de igualdad. Aldao y Clérico (2019) distinguen diferentes fórmulas para definir dicho principio. La menos exigente y más tradicional es la igualdad formal, que surge de una clasificación realizada por el legislador y permite distinguir categorías formales pero no examina las razones ni los criterios de construcción de dichas categorías. Un segundo tipo de fórmula es aquella que se pregunta por la legitimidad de los criterios de clasificación y las razones en las que se basa la selección. Se trata, en este caso, de la igualdad jurídica material. Finalmente, los autores definen la perspectiva de la igualdad como redistribución y reconocimiento que apunta a evidenciar desigualdades de orden material y simbólico, por lo cual la igualdad no refiere a un presupuesto sino a una meta a alcanzar ${ }^{1}$.

Aplicar al análisis de la educación -como objeto de derecho- el principio de igualdad a la luz de sus diferentes definiciones, resulta una tarea intelectual necesaria dado que este principio se encuentra en la base de los derechos humanos fundamentales. Por consiguiente, cabría preguntarse a la luz del panorama de emergencia generado por la pandemia del Covid-19, que supuso el cierre compulsivo de sistemas e instituciones educativas, en qué medida se vio afectada la igualdad educativa: ¿cuán factible es el reemplazo de la enseñanza de contenidos diseñada para la educación presencial por programas de educación a distancia? Sobre todo cuando ello es decido de manera abrupta. Aquí podría identificarse un punto de incertidumbre en relación con la cuestión sobre derecho a qué exactamente es el derecho a la educación cuando es ejercido en estas condiciones de emergencia.

En esta dimensión de análisis es pertinente incluir el principio de equidad como complementario al de igualdad de modo tal que pueda pensarse en el derecho a la educación como un derecho que incluye las necesidades educativas de los sectores más desventajados en términos materiales, culturales o simbólicos. De manera congruente con la fórmula de igualdad de reconocimiento y redistribución, la equidad implica tener en cuenta las desigualdades que son encubiertas cuando el prisma se limita a la igualdad formal. Sólo a través de la redistribución proporcional de los recursos educativos en función de las necesidades de los sectores más pobres, se lograría la promoción de una justicia distributiva en educación (Bolívar, 2005).

Un aspecto central en esta tarea de conceptualización sobre el contenido y el alcance del derecho a la educación, podría estar dado por el significado de los procesos de aprendizaje de los estudiantes (Darling-Hammond, 2001; McCowan, 2013). Ello permitiría evaluar no sólo el desarrollo humano de cada estudiante sino también cómo esos aprendizajes favorecen el desarrollo social de las personas, en cada contexto histórico y cultural en

\footnotetext{
${ }^{1}$ En este punto resulta válido recuperar el análisis de Bobbio (1993) cuando analiza el principio de igualdad en comparación con otros como la libertad. La igualdad hace referencia a una relación, para cuya interpretación concreta el autor brinda el ejemplo de las expresiones "esta persona es libre" y "esta persona es igual"; la segunda exige preguntarse ¿igual a quién o a qué? A partir de ello, Bolívar (2005) distingue cuatro combinaciones posibles en materia de igualdad educativa; dos de ellas serían elitistas -igualdad para algunos en todo, igualdad entre algunos en algo-, mientras que las otras dos no: igualdad entre todos en todo -que sería la postura más radical-, e igualdad entre todos en algo. Esta última sería la más asequible en materia educativa, pero es la que plantea la pregunta: ¿en qué serían iguales las personas en términos de la educación como derecho? Las opciones de respuestas son variadas: en el acceso a los niveles educativos, en términos de contenidos curriculares a aprender, en el goce de los mismos recursos educativos, en la conformación de los grupos de aprendizaje, entre otras (Ruiz, 2020).
} 
particular. Colocar el foco en los procesos desde el punto de vista del contenido del derecho a la educación, supone promover el carácter activo de la enseñanza como uno de los mayores potenciales de la escolarización (Dewey, 1970). Cuestiones y procesos tales como la indagación, el diálogo, la comprensión lectora, la deliberación y la exploración de la argumentación para analizar las diferencias en términos de perspectivas analíticas, son algunos de los procesos educacionales que podrían ser incluidos desde esta perspectiva. En tal sentido, la promoción de estas competencias podría ser congruente con el planteo de Guttman (2001) en relación con pensar a la educación escolar como un ideal político y educativo ${ }^{2}$.

En este punto cobra relevancia también la discusión en torno a los límites de la educación formal en las sociedades contemporáneas, dado que los aprendizajes pueden acontecer en múltiples ámbitos diferentes a los escolares. Asimismo, ello puede a su vez representar para muchos la posibilidad de continuar la escolarización formal o bien abandonarla, lo cual es en sí mismo un desafío para los Estados en materia de las obligaciones prestacionales que tienen respecto de garantizar el ejercicio de este derecho. Por ello, cabría incorporar una escala de análisis al catálogo de preguntas que demarcan la conceptualización del derecho a la educación (Ruiz, 2020). Esta escala permitiría determinar en buena medida qué se entiende por derecho a la educación cuando se lo invoca.

Con esto se quiere destacar que no es suficiente pensar el derecho a la educación solamente a partir los estándares internacionales y de los contenidos básicos para regular los derechos garantizados a todos los seres humanos, ni tampoco alcanza con analizar el cumplimiento de las obligaciones que dichos derechos generan para los Estados hacia sus habitantes. Se requiere asimismo identificar en función de dichos estándares internacionales, derivados del derecho internacional de los derechos humanos, las implicancias formativas que en cada sociedad tiene el ejercicio de este derecho (Ruiz, 2020). Para lo cual, el principio de igualdad de reconocimiento y redistribución, así como el de equidad en la definición de las prestaciones educacionales pueden proveer indicadores válidos para la medición del derecho a la educación, en cada contexto socio-histórico particular. Podría pensarse que, en un plano estrictamente pedagógico, que atañe a la formación de las personas en función de las oportunidades educativas que tienen, el derecho a la educación adquiere diferentes significados de acuerdo con el nivel educativo que se analice y considere.

\section{El cierre de la educación formal como medida sanitaria para el control de la pandemia}

Al igual que un siglo atrás, ante la pandemia del Covid-19 los llamados recursos no farmacéuticos se han impuesto: el encierro de la población (sana en este caso) en sus

\footnotetext{
${ }^{2}$ No obstante, es necesario advertir que otorgar centralidad a los procesos educativos no supone descuidar o negar la promoción de resultados o de estándares de formación sin que ello necesariamente implique a su vez estandarizar a los estudiantes, ni menos aun desatender la formación curricular disciplinar. Al contrario, cada proceso educativo podría ser desarrollado a través de estrategias de enseñanza que analicen y cuestionen los contenidos que provienen de la lengua, la matemática, las ciencias sociales, las ciencias naturales, las artes, y la educación física, de acuerdo con las etapas evolutivas y los contextos culturales en los cuales se desarrolla la educación formal (Ruiz, 2020).
} 
hogares, y la higiene con agua y jabón. A pesar de esta semejanza con el pasado, se ha producido una situación excepcional: el cierre de las clases presenciales en todos los niveles educativos en la amplia mayoría de los países del mundo, lo cual se basa en la creencia de que de esta forma se reduciría el contacto entre las personas y así se interrumpiría la transmisión del virus (Viner et al., 2020) ${ }^{3}$. Se trata de una situación crítica de alcance global que no tiene precedentes desde que la obligatoriedad escolar ha sido instrumentada gradualmente en el mundo occidental desde el siglo XIX. Recordemos que el aula y la institución educativa formal han sido diseñadas como espacio material y estructura comunicativa, o sea como un espacio específicamente concebido para la escolarización masiva. El aula de la educación moderna se construyó además sobre la idea del para todos y para cada uno, la cual nos remite al principio de igualdad. Las políticas dispuestas a partir de marzo de 2020 han modificado estas características básicas ya que han apuntado a mantener la continuidad pedagógica de la educación formal a través de plataformas digitales. Así, se generó una situación en la cual se evidencia una separación del espacio comunicativo respecto del material. La enseñanza se ha separado de la copresencia en las aulas e instituciones educativas, y los hogares se han transformado en espacios de trabajo escolar.

Pueden identificarse algunos antecedentes similares que han afectado a regiones o países del mundo en anteriores epidemias (de cólera, tifus) o pandemias (como la de gripe de 1918-1919, o la de malaria, entre otras), en los cuales se implementaron diferentes cierres escolares denominados reactivos o proactivos (Jackson et al., 2013). En los primeros se dispone el cierre por haberse identificado personas (estudiantes, docentes) infectados en la institución escolar; en los segundos, en cambio, con el cierre del establecimiento educativo se trata de evitar la propagación de enfermedades, lo cual constituye en sí mismo una de las herramientas más disruptivas aplicadas a la educación formal. Cuando ello afecta a la educación no obligatoria (nivel secundario superior, en algunos países, y educación universitaria) abarca al conjunto de la población joven adulta también, y con ello restringe de manera notoria el acceso a la formación de la mayoría de la población de un país, por establecerse un distanciamiento social forzoso como mecanismo de aislamiento para prevenir la expansión de la pandemia.

Este último tipo de cierre, uno de los mecanismos más poderosos de intervención no farmacéutica, ha sido el dispuesto por los países a partir de marzo de 2020 (CouzinFrankel, 2020). Esta decisión no sólo restringe el acceso a la educación formal sino que además posee el riesgo de afectar a la nutrición de los estudiantes debido a que en muchos países la población escolar recibe alimentación diaria en las instituciones educativas y su salud puede verse afectada debido al aislamiento forzado dispuesto a través de los cierres proactivos de los sistemas escolares de alcance masivo, más aún cuando también se encuentra afectada la cadena de distribución de alimentos por el cierre de otros sectores y servicios.

Son escasas las investigaciones médicas que han analizado los efectos del cierre de establecimientos de educación formal por la expansión de pandemias; de igual modo hay poco consenso acerca de los beneficios de estas medidas no farmacéuticas. Es más, también se pone en discusión sus costos, en términos de la salud mental de las personas y en los

${ }^{3}$ Agradezco los datos y las fuentes -que refieren a investigaciones médicas-provistas por Luis Miguel Lázaro Lorente, las cuales han sido utilizadas en esta sección. 
efectos nocivos para la alimentación de los estudiantes que asisten a la escuela y que reciben su ración diaria de comida. Los análisis de bases de datos de trabajos publicados sobre el tema y los estudios de modelización sugieren que su influjo en el control de la pandemia Covid-19 podría oscilar entre un 2 y un $4 \%$ de reducción de la mortalidad (Viner et al., 2020). Es así que se vería afectado no sólo el derecho a la educación (tanto por no asistir a las instituciones sino porque no se posee la infraestructura necesaria para suplir ello a través de programas de educación a distancia) sino también la economía familiar, la salud de la población infantil y joven, todo lo cual sería más notorio en los sectores más empobrecidos. Asimismo, esta decisión tiene implicancias para el futuro ya que ante una situación de pandemia cabría planificar con mucho cuidado la reapertura de los establecimientos escolares en función de los resguardos de bioseguridad necesarios para disponer el retorno de la comunidad educativa a las instituciones y a sus espacios físicos que son comunes para estudiantes, docentes y personal administrativo.

Ante este panorama los gobiernos decidieron apelar a modalidades de enseñanza a distancia para evitar que se interrumpan los ciclos escolares, con las diferencias entre los hemisferios norte y sur de acuerdo con esa época del año. ${ }^{4}$ En un informe del día 6 de abril de 2020, los responsables de UNESCO admitieron que se trataba de una crisis sin precedentes en la historia de la educación escolar, tal como puede observarse en el mapa de la figura 1 que refiere al período de máxima cantidad de países con cierre de escuelas.

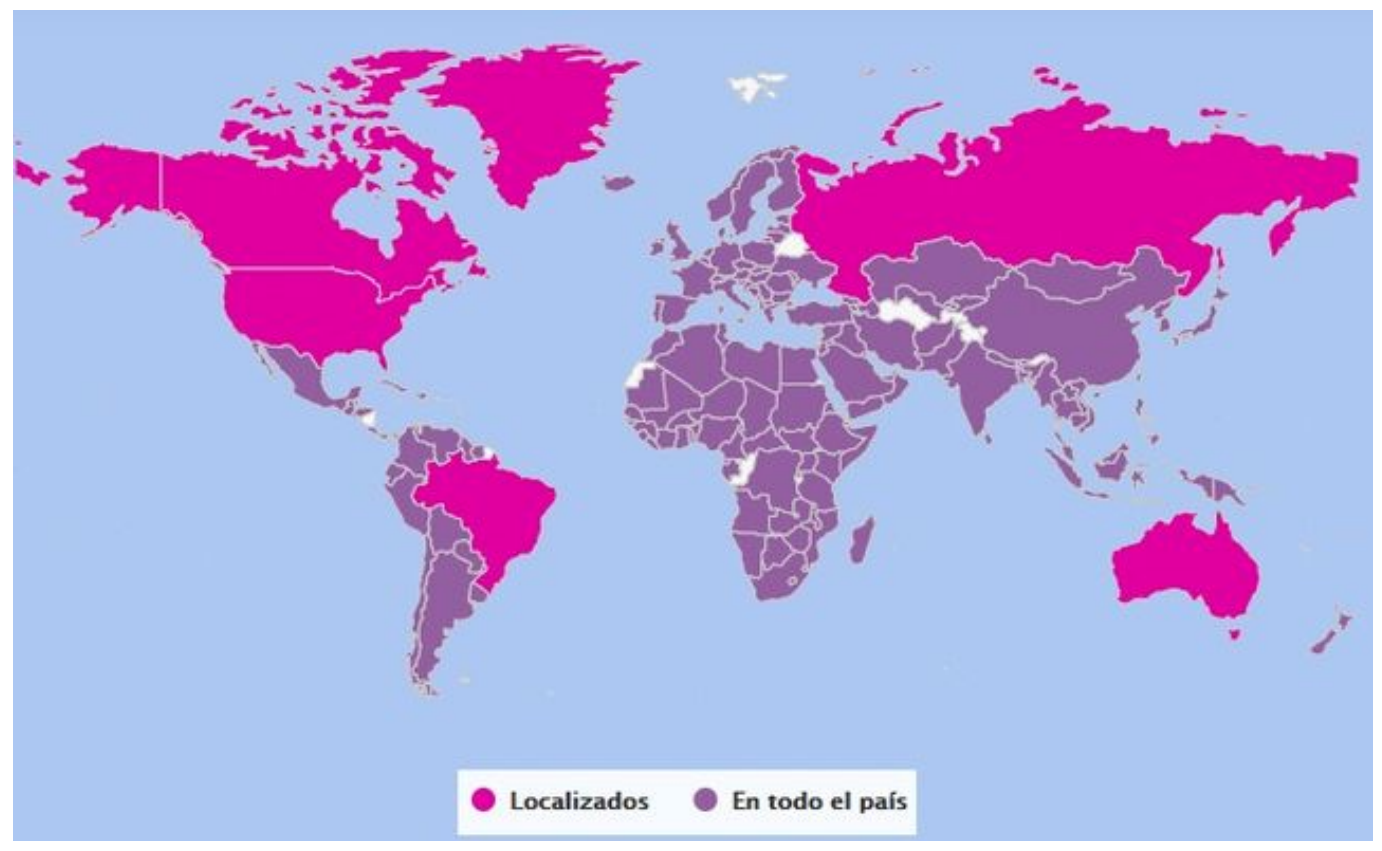

Figura 1. Mapa de países afectados por el cierre de escuelas, según el alcance del cierre (localizado o en todo el país). Situación en abril de 2020

Fuente: Informe interrupción educativa y respuesta al COVID-19, UNESCO (2020).

\footnotetext{
${ }^{4}$ Mientras en el hemisferio norte se promediaba la segunda parte del año académico iniciado en septiembre de 2019, en el hemisferio sur se comenzaba la primera parte del año académico 2020. En cada caso, ello involucraba decisiones de acreditación de cursos y de programación didáctica diferentes pero igualmente importantes para garantizar los procesos de aprendizaje así como los de evaluación y acreditación de saberes y cursos.
} 
En el mapa se distinguen con diferentes colores los sistemas escolares cerrados en todo un país, de aquellos países en los cuales se han cerrado los sistemas en algunas regiones o bien en algunos niveles educativos. En dicho informe, que se ha actualizado de forma periódica, la UNESCO señala que durante las semanas posteriores al inicio de la interrupción de las clases presenciales la cantidad de personas que había sido alcanzada por el cierre de los sistemas escolares creció de forma exponencial: el 4 de marzo había 300 millones de estudiantes impactados en una veintena de países (desde la educación inicial hasta la superior); en menos de un mes esa cifra ascendía a más de 1.500 millones, en 188 países. Lo cual constituía el 90 \% de la población estudiantil del mundo; a los que se sumaban 60 millones de profesores ${ }^{5}$. En América Latina la suspensión de las actividades presenciales se dispuso en primer término en Colombia y Perú el 12 de marzo de 2020 y en los 6 días posteriores alcanzó a casi la totalidad de estudiantes de todos los niveles educativos de la región. En el caso de la educación superior en particular, las estimaciones de UNESCO indican que en América Latina unos 23,4 millones de estudiantes y 1,4 millones de profesores estarían afectados por este cierre, lo cual representa un $98 \%$ de la población universitaria de la región (IESALC, 2020).

Con este contexto, los gobiernos han convocado a las instituciones educativas de todos los niveles a continuar con las tareas de enseñanza a través de la educación a distancia para así garantizar la continuidad de los estudios sin que se perdieran años, cursos, cuatrimestres o semestres según sea el caso. Esta convocatoria ha generado diferentes consecuencias tanto entre el profesorado, como entre los estudiantes quienes deben aprender en sus hogares contenidos que habían sido planificados para clases presenciales. Ahora bien, cabe preguntarse cuánto ha sido afectado el derecho a la educación.

\section{Discusión: ¿Qué consecuencias tiene la educación través de plataformas digitales para el ejercicio del derecho a la educación?}

La adopción generalizada y vertiginosa de la educación a distancia como forma de mantener los estudios, a través de plataformas digitales, ha sido una de las decisiones más generalizadas por gobiernos y autoridades educativas. Así, la virtualización de las clases presenciales, tanto por su carácter masivo y compulsivo como por la forma de comunicación y trabajo con los estudiantes, encontró diversos problemas técnicos y generó desafíos para rediseñar -en la acción- las estrategias didácticas de docentes y el rol de los estudiantes (y el de los padres en el caso de la educación inicial-infantil y primaria).

Aquí resulta válido hacer una somera referencia a la aplicación de tecnologías digitales a la educación. Las tecnologías en su conjunto ocupan un lugar central en las sociedades contemporáneas, en diferentes dimensiones, y el desarrollo de plataformas digitales es

\footnotetext{
${ }^{5}$ El registro periódico de este recurso disponible en el sitio de UNESCO toma como fecha de inicio el día 16/02/2020 con el cierre dispuesto por China primero y seguido por Mongolia unos días después. Hacia el 31/03/2020 se alcanzaba el máximo valor de estudiantes afectados en el mundo por el cierre escolar (91,3\%); el día 23/04/2020 descendía dicho valor al $89 \%$; el 01/05/2020 se ubicaba en 73,5\%; y se mantenía alto durante las semanas siguientes $(68,5 \%$ hacia el 25/05/2020). Véase

https://en.unesco.org/covid 19/educationresponse
} 
muy variado (entre muchos otros pueden enumerarse: entornos virtuales, redes sociales, plataformas de servicios y de productos). Mucho de este desarrollo ha impactado en los diferentes niveles de la educación formal, aunque con características muy distintas en función de los grados de desarrollo socio-económico de los países o bien si corresponde a la educación obligatoria, o a la formación en los campos profesionales de la educación superior. Así, en las últimas décadas ha sido cada vez mayor la progresiva introducción de distintos modos de enseñanza que han flexibilizado los modelos de pedagogía -sobre todo- universitaria (blended-learning, e-learning, m-learning, adaptative learning, entre otros). Por otra parte, se destaca la importancia adquirida por la formación permanente como modelo para el profesorado de todos los niveles escolares. No se trata de micro habilidades instrumentales, sino que ello se entrecruza con un ejercicio reflexivo sobre el rol docente, las peculiaridades de los estudiantes, y las condiciones institucionales, entre otros aspectos (Cardona, 2017). Algunos autores señalan la tendencia a la convergencia entre las conexiones rizomáticas de las plataformas y la analítica de datos, así como la dataficación y la digitalización de la información, todo lo cual da lugar a plataformas cada vez más similares (Lion, 2019; Williamson, 2018). Ello genera cada vez más nuevos tipos de mediaciones en la formación de los sujetos de la educación.

En consonancia con lo precedente, muchas investigaciones sobre la educación a distancia a través de plataformas digitales han sostenido la necesidad de cambiar las concepciones de enseñanza de los contenidos escolares y de la pedagogía universitaria así como de su aprendizaje (De Pablo Pons, 2009; Litwin, 2000; Maggio, 2018). El principal cambio estaría dado por las características del espacio virtual, en el cual no aplican las nociones de lugar y tiempo escolar propias de la educación presencial (con horarios de entrada, en días específicos, por turnos, en espacios arquitectónicos particulares a los cuales asisten estudiantes y docentes). El espacio virtual y sus recursos modifican dichos esquemas, y suponen diferentes enfoques de la didáctica en los cuales lo grupal puede tener centralidad, pero la socialización vivencial no acontece como lo hace en las clases presenciales, las cuales constituyen ambientes multisensoriales (De Pablo Pons, 2006; Lion, Mansur y Lombardo, 2015). No es este el lugar para analizar las posibilidades y limitaciones de la educación a distancia en sí misma, aunque cabría destacar que la presencialidad no puede ser sustituida ni ello debería ser el propósito perseguido cuando se instrumenta un programa de educación a distancia. Lo destacable en el contexto contemporáneo generado por la pandemia del Covid-19 es cómo algunas de las herramientas de la educación a distancia han sido adoptadas e implementadas con celeridad extraordinaria en todos los niveles escolares, en la mayoría de los países del mundo. Cabe señalar que estas herramientas contienen una lógica individual que requiere de una formación específica del profesorado para poder utilizarlas de manera que favorezca aprendizajes y garantice el derecho a la educación. La tarea docente consiste en promover la motivación y la autonomía entre los estudiantes y sería riesgoso que ello se torne en condiciones previas exigidas a los estudiantes para afrontar la educación en plataformas digitales.

Es válido advertir que entre las evidencias recolectadas por la investigación en este campo se ha encontrado que la tecnología digital (aplicada a la enseñanza) a veces ha tenido poco impacto en el aprendizaje, principalmente porque no alcanza con dotar de ordenadores la escuela, sino que es preciso darle un sentido pedagógico a esta infraestructura y a estos recursos informáticos (Cobo, 2016). También se destaca la necesidad de promover diversas habilidades (pensamiento adaptativo, pensamiento computacional, alfabetización 
mediática, colaboración virtual, por mencionar algunas) que son factibles de favorecer en aulas equipadas y con usos intensivos por parte del cuerpo docente de las tecnologías; especialmente en ambientes de alta disposición tecnológica (Maggio, 2012). Ello no refiere solamente de la cantidad de computadoras en las instituciones educativas y en las aulas sino además a la presencia de teléfonos celulares, aulas móviles y de estudiantes acostumbrados a una conexión permanente. Este contexto genera consecuentemente un reto tanto en la formación inicial cuanto en el desarrollo profesional docente, en la medida en que surge la necesidad de promover la competencia digital docente (Lion, 2019; Williamson, 2018). Así es que resulta necesario no sólo entender cómo usar las tecnologías aplicadas a la educación, sino comprender su impacto potencial en los procesos de enseñanza y de aprendizaje que pueden generarse (o bien limitarse) a través de las plataformas digitales.

Ahora bien, dichas plataformas digitales contemplan múltiples herramientas para el desarrollo de diversas estrategias de enseñanza ${ }^{6}$. El espacio virtual genera condiciones diferentes a las clases presenciales y en tal sentido da lugar a intercambios caracterizados por la mediación de la infraestructura tecnológica que, a la vez, posibilita tiempos de elaboración asociados con esta mediación (De Pablo Pons, 2006). En cualquier caso, se destaca siempre el rol docente en las tutorías y en el seguimiento de estudiantes, así como los materiales que se les ofrece para que puedan lograr producir un trabajo de elaboración individual o grupal. En general, se suele recomendar la tutoría y el seguimiento de los procesos de aprendizaje en diferentes instancias. Tareas que requieren otra forma de administrar las intervenciones docentes, una vez que se implementan las estrategias de enseñanza a través de las plataformas digitales, con una finalidad orientadora y de guía para que el estudiante trabaje sobre su propio proceso de aprendizaje (Lion, Mansur y Lombardo, 2015; Maggio, 2018). Todo ello demuestra la importancia que poseen las instancias de programación didáctica y también las condiciones de infraestructura y de composición de los grupos de estudiantes.

La masividad y la celeridad de la adopción de la educación a distancia en este período de pandemia no parecen reconocer estos condicionantes y pueden generar decisiones didácticas que surgen de la planificación atemporal o sobre el mismo período de ejecución de la enseñanza. Algunos autores (Lion, Mansur y Lombardo, 2015; Maggio, 2018; Williamson, 2018) advierten que la educación virtual con acciones que promueven la descentralización, la multitarea, la convergencia y lo que se denomina lifestreaming, atentan contra la atención del estudiantado, a la vez que compiten con una serie de estímulos por las redes que son inmanejables para los docentes (los estudiantes pueden aparecer conectados pero puede que en realidad en ese momento realicen otra acción a través del chat - por ejemplo- que desvirtúa la propuesta didáctica implementada).

\footnotetext{
${ }^{6}$ Es posible trabajar a partir de videos y recursos bibliográficos, presentaciones en power point y podcast, a partir de los cuales se pueden instrumentar actividades individuales y grupales en función de los propósitos de enseñanza. El foro se destaca como una herramienta útil para generar no sólo el intercambio a partir de preguntas y respuestas, sino para promover la orientación y la tutoría que debería desplegarse en los tiempos particulares que posee cada estudiante. Por otra parte, el uso de herramientas de videoconferencia no es lo recomendado y es incluso un error cuando se trata de grupos masivos como ocurre en muchas instituciones universitarias de América Latina. En general se lo considera un resabio que trata de trasladar al espacio virtual la modalidad presencial. Esta enumeración no es exhaustiva, al contrario, como toda tecnología educativa tradicional o contemporánea es funcional al contenido de la enseñanza y a las estrategias programadas y desplegadas por docentes en los grupos de aprendizaje.
} 
A esto cabría agregar el contexto de aplicación de estas respuestas (por parte de las autoridades educativas, así como por parte de las instituciones), el cual se caracteriza por la incertidumbre y el encierro forzado y abrupto de la población, que constituyen factores de estrés inconmensurables que redundan en el propio desarrollo educativo y en el trabajo intelectual con él asociado. Ello no necesariamente contribuye a la mejor predisposición para el aprendizaje de contenidos de la educación formal (en cualquiera de sus niveles) ni tampoco al uso eficiente y autorregulado del tiempo de estudio, que sería una de las características más favorables que la educación virtual tendría en cualquier programa educativo específicamente diseñado como tal.

Finalmente, un aspecto sumamente importante a considerar es el acceso de la población a los recursos que brindan las tecnologías aplicadas a la educación a distancia. Burbules y Callister (2001) diferencian entre un acceso instrumental y un acceso real. El primero se vincula con la posibilidad de contar con la infraestructura necesaria (equipamiento, conectividad) para poder acceder al contenido educativo. El segundo tipo de acceso -el real- refiere a la posibilidad de una apropiación relevante de las tecnologías, en términos de formación de las personas, un acceso que debería ser equitativo, desde el enfoque del derecho a la educación, en la medida que favorezca aprendizajes equivalentes entre los estudiantes. Aquí cabría tener presente además que en muchos contextos-como los países subdesarrollados- el acceso a ordenadores no ocurre en el hogar y suele suplirse, al menos parcialmente, con el acceso a estos dispositivos en las instituciones educativas o bien con dispositivos de telefonía celular/móvil.

\section{Conclusiones: implicancias y escenarios para el ejercicio del derecho a la educación}

Algunas voces interpretan que esta crisis supondría un cambio a largo plazo en los sistemas masivos de escolarización ya que se ha generado un desafío en la medida en que la educación formal a partir de este cambio excedería a la clase presencial (Dans, 2020). En estos escenarios los cambios instrumentados parecen no ser una opción que podría desmantelarse una vez concluido el aislamiento social obligatorio sino una decisión de política educativa a largo plazo. En consecuencia, algunas formas de enseñanza no tendrían lugar en los nuevos espacios educativos virtuales. La selección de la información estaría limitada por el contexto en los cuales se instrumentaría la enseñanza online, que fomentaría el trabajo grupal, la evaluación de pares, y que repensaría el rol docente quien además de tutor debería incrementar su dedicación a la planificación de actividades para luego moderar discusiones entre estudiantes que asumirían centralidad en fórums dando lugar así a una mayor adaptación de la información (más que contenidos de enseñanza) a las características del estudiantado (Dans, 2020). Todo ello bajo el argumento que supone que habría un mayor activismo pedagógico por parte de estudiantes, quienes a la vez serían pares en comunidades de aprendizaje las cuales romperían con el tradicionalismo de la educación formal.

Sin embargo, según un estudio de IESALC (2020), los cambios instrumentados en el corto plazo de semanas durante marzo y abril de 2020 para instrumentar la educación a distancia (a través de diversas plataformas digitales) no han sido recibidos de forma positiva en América Latina. Ello por muchas razones, entre ellas se mencionan las expectativas de los estudiantes en relación con los estudios presenciales que involucran factores sociales y de 
experiencia de vida que no están presentes en la educación a distancia. Esta última además supone mayor disciplina y exigencia para los estudiantes más aún cuando deben tratar de acceder a contenidos que fueron planificados para ser enseñados en forma presencial. A su vez, para los estudiantes de sectores vulnerables la asistencia a la educación presencial brinda oportunidades de interacción en ambientes (bibliotecas, laboratorios, clases que facilitan la socialización con pares de diferentes orígenes o antecedentes sociales y culturales) que están ausentes en sus contextos familiares.

El informe también indica que estas decisiones han afectado al profesorado, en diferentes niveles de su desarrollo profesional. La precariedad de los recursos institucionales y la falta de tiempo para la planificación de la enseñanza a través de plataformas digitales (desconocidas por los docentes) han generado situaciones muy diversas en función de las instituciones educativas, de los estratos sociales y también en relación con la disposición de herramientas y recursos, así como de acceso a la capacitación. Al estar abrumado por el tiempo, el profesorado de la región se ha visto obligado a responder con múltiples propuestas que son planificadas en el mismo período en que son ejecutadas (IESALC, 2020). Es decir, se subvierten así los principios de la planificación y programación didáctica y ello redunda en las posibilidades de favorecer aprendizajes equivalentes entre estudiantes que evidencian también diferentes posibilidades de acceso a las plataformas digitales desde sus hogares.

Sin duda, el esfuerzo realizado por el profesorado es loable y extraordinario, y ha generado propuestas creativas en algunos casos. Es más, ha sido muy honorable la respuesta y compromiso del profesorado de todos los niveles educativos para sostener la continuidad pedagógica. No obstante, resulta válido cuestionar si se ha respetado el principio de igualdad y el de equidad en los que se sostiene el derecho a la educación. La educación a distancia tiene múltiples beneficios y ha probado ser eficaz sobre todo en la formación de posgrado universitario, y también en la formación profesional continua, ya que la infraestructura tecnológica y la disponibilidad de acceso a estas plataformas es para grupos más reducidos y en programas que son diseñados específicamente para ser desarrollados a través de dichas plataformas (IESALC, 2020). Diferente es el caso de las decisiones adoptadas por los gobernantes y por las instituciones educativas ya que obligaron a realizar esfuerzos de conversión apresurada de aquello que había sido diseñado para la enseñanza presencial a ser procesado a través de plataformas digitales (la cuales no estaban generalizadas en el conjunto de las instituciones educativas). ${ }^{7}$

Algunos autores pregonan que se debe profundizar la investigación sobre la incidencia del cierre de los establecimientos educativos en la declinación de los contagios ya que aún resulta escasa la evidencia empírica, sobre todo aquella que analiza lo ocurrido luego de la

\footnotetext{
7 Un aporte para el diseño de opciones de salida a esta situación está dado por un informe de diagnóstico publicado por la Fundación COTEC (2020). Dicho trabajo, si bien toma datos de Corea del Sur, China, Japón, Suecia, Singapur y Egipto, se centra en el caso español. Realiza, además de un riguroso diagnóstico de las respuestas ante la pandemia (por parte de los países mencionados), una propuesta de cinco escenarios de actuación, que refieren sobre todo para España y otros países del hemisferio boreal debido a las diferencias de los calendarios escolares y académicos entre los hemisferios norte y sur. Este documento constituye un aporte valioso para instrumentar medidas de acción (en términos de provisión de recursos y medidas sanitarias anticipatorias así como curriculares y de evaluación de aprendizajes), aunque su ámbito de aplicación no abarca al conjunto de los países del mundo. Ello principalmente debido a los diferentes niveles de desarrollo socioeconómico, ya que los problemas identificados, tanto sanitarios como pedagógicos (que son comunes al conjunto de países en el contexto de emergencia generado por el Covid-19), se agudizan en los casos nacionales en los cuales existen problemas económicos y sociales de profunda desigualdad que agravan a los primeros.
} 
reapertura de los establecimientos escolares (Jackson et al., 2013). Es más, algunos autores (Viner et al., 2020) sostienen que el cierre compulsivo de forma proactiva -de los establecimientos de educación formal- puede tener altos costos incluso para el sistema de salud en la medida en que afecta a profesionales quienes no pueden asumir sus responsabilidades -en las instituciones hospitalarias- debido a sus compromisos parentales (al no tener formas alternativas de cuidar a sus propios hijos y tener que quedarse también en sus hogares). Además, el riesgo que se advierte en algunas investigaciones (Cobo, 2019; Cohen, 2017) es que el abandono escolar se incremente, lo cual sería más notorio en la educación superior y en las regiones empobrecidas como América Latina. Ello por razones económicas (la salida de la crisis será traumática en términos del aumento de la pobreza y del desempleo) y también por razones pedagógicas. Estas últimas refieren a que los estudiantes al no poder acceder al conjunto de contenidos distribuidos en las plataformas digitales, y al no conseguir comprender las tareas que allí fueron dispuestas por el profesorado, se desconecten del ritmo académico que implica la educación formal y más aún en el nivel superior. A su vez, el profesorado también puede ser afectado en múltiples formas. ¿Qué sucedería si se generaliza la idea que la presencialidad puede ser reemplazada por la educación a distancia? ¿Cómo se instrumentaría la educación formal si se asume que es posible que la enseñanza pueda acontecer a través de plataformas digitales (comercializadas en un nuevo tipo de cuasimercado educativo)?

La escala y la celeridad del cierre proactivo de los sistemas e instituciones educativas, que afecta a los niveles obligatorios y superiores de la educación formal no tiene precedentes y no resulta claro cuánto se puede prolongar ni qué decisiones se pueden tomar para garantizar la continuidad de los estudios y su acreditación. Esto a su vez en cada nivel escolar implica decisiones muy serias en relación con el acceso al saber y al conocimiento de forma de ejercer el derecho a la educación. Las consecuencias de una restricción de largo tiempo al sistema educativo y su reemplazo compulsivo por programas de educación a distancia, con escaso margen de planificación, redundará además en la salud de la población escolar joven y adulta, y a la vez también tendrá impactos a largo plazo en los resultados educativos tales como el nivel de formación, los niveles de ingresos futuros (tasas de retornos), la productividad de las sociedades, entre otros indicadores de desarrollo social y económicos de los países. Por último, hay que destacar que la educación a distancia puede incrementar la desigualdad educativa ya existente dado que pone en evidencia brechas de diferentes tipos a las visibles en la presencialidad: brechas de infraestructura, de conectividad, de posibilidades de usos formativos por parte de docentes y estudiantes de los recursos tecnológicos. A ello cabría sumar la potencial promoción de una concepción comercial de la educación en relación con el consumo de software (en lugar de los contenidos curriculares). La educación formal cumple muchas funciones más allá de transmitir conocimientos, hay que considerarla un espacio de conversación, de trabajo para lo común y lo individual, bajo el principio de igualdad. Por lo expuesto, es importante reconocer que el mayor riesgo es que no se contemplen profundamente las condiciones de emergencias (sanitarias, sociales, psicológicas y tecnológicas) generadas por la pandemia del Covid-19, ya que ello afectará el ejercicio del derecho a la educación y sus implicancias formativas para el desarrollo humano. 


\section{Referencias}

Aldao, M. y Clérico, L. (2019). La igualdad "reformada”: La igualdad “des-marcada”. En N. Cardinaux y L. Clérico (Comps.), Formación de jueces: Su adecuación a un modelo de sociedad igualitaria (pp. 193-216). EUDEBA.

Altbach, P. y Kelly, G. (1986). New approaches to comparative education. The University of Chicago Press.

Bobbio. N. (1993). Igualdad y libertad. Paidós.

Bolívar, A. (2005). Equidad educativa y teorías de la justicia. REICE. Revista Iberoamericana sobre Calidad, Eficacia y Cambio en Educación, 3(2), 42-69.

Burbules, N. y Callister, T. (2001). Educación: Riesgos y promesas de las nuevas tecnologías. Granica.

Cardona, D. (2017). Reseña. Horizon report 2017 higher education. https://doi.org/10.5209/TEKN.58102

Cobo, C. (2016). La innovación pendiente. Reflexiones (y provocaciones) sobre educación, tecnología y conocimiento. Penguin Random House.

Cobo, C. (2019). Acepto las condiciones. Usos y abusos de las tecnologías digitales. Fundación Santillana.

Cohen, A. (2017). Analysis of student activity in web-supported courses as a tool for predicting dropout. Educational Technology Research and Development, 65, 1-20. https://doi.org/10.1007/s 1 1423-017-9524-3

Couzin-Frankel, J. (2020). Does closing schools slow the spread of coronavirus? Past outbreaks provide clues. Science, $0-84$. https://doi.org/10.1126/science.abb6686

Dans, E. (13 de abril de 2020). The coronavirus pandemic has unleashed a revolution in education: From now on, blended learning will be the benchmark. Revista Forbes. https://www.forbes.com/sites/enriquedans/2020/04/13/the-coronavirus-pandemic-hasunleashed-a-revolution-in-education-from-now-on-blended-learning-will-be-thebenchmark/\#44ecfeb6536f

Darling-Hammond, L. (2001). El derecho de aprender. Crear buenas escuelas para todos. Ariel.

Dewey, J. (1970). Democracia y educación. Losada.

Fundación COTEC. (2020). COVID-19 y educación: Problemas, respuestas y escenarios. https://online.flippingbook.com/view/967738/12/

Guttman, A. (2001). La educación democrática. Una teoría política de la educación. Paidós.

IESALC. (2020). COVID-19 y educación superior. De los efectos inmediatos al día después. Análisis de impactos, respuestas políticas y recomendaciones. http://www.iesalc.unesco.org/2020/04/14/iesalc-insta-a-los-estados-a-asegurar-elderecho-a-la-educacion-superior-en-igualdad-de-oportunidades-ante-el-covid-19/

Jackson C., Vynnycky E., Hawker J., Olowokure, B. y Mangtani, P. (2013). School closures and influenza: Systematic review of epidemiological studies. British Medical Journal Open, 3, e002 149. https://doi.org/10.1136/bmjopen-2012-002149

Lion, C. (2019). Los desafíos y oportunidades de incluir tecnologías en las prácticas educativas. Análisis de casos inspiradores. IIPE.

Lion, C., Mansur, A. y Lombardo, C. (2015). Perspectivas y constructos para una educación a distancia re-concebida. Revista del Instituto de Investigaciones en Ciencias de la Educación, 37, 101-117.

Litwin, E. (Comp.). (2000). La educación a distancia. Amorrortu. 
Maggio, M. (2012). Enriquecer la enseñanza. Los ambientes con alta disposición tecnológica como oportunidad. Paidós.

Maggio, M. (2018). Reinventar la clase en la universidad. Paidós.

McCowan, T. (2013). Education as a human right. Principles for a universal entitlement to learning. Bloomsbury.

Meyer, J. y Ramírez, F. (2010). La educación en la sociedad mundial. Teoría institucional y agenda de investigación de los sistemas educativos contemporáneos. Octaedro.

Pablo Pons, J. de (2006). El marco del impacto de las tecnologías de la información. Herramientas conceptuales para interpretar la mediación tecnológica educativa. Revista Telos, 67, 31-56.

Pablo Pons, J. de (Coord.). (2009). Tecnología educativa, la formación del profesorado en la era de Internet. Aljibe.

Ruiz, G. (2020). El derecho a la educación: Definiciones, normativas y políticas públicas revisadas. EUDEBA.

Tomasevski, K. (2001). Human rights obligations: Making education available, accessible, acceptable and adaptable. Novum Grafiska AB.

Tröhler, D. y Lenz, D. (Comps.). (2015). Trayectorias del desarrollo de los sistemas educativos modernos. Entre lo nacional y lo global. Octaedro.

UNESCO. (2020). Interrupción educativa y respuesta al COVID-19. https://es.unesco.org/covid 19/educationresponse

Viner, R. M., Russell, S. J., Croker, H., Packer, J., Ward, J., Stansfield, C. ... y Booy, T. (2020). School closure and management practices during coronavirus outbreaks including COVID19: A rapid systematic review. The Lancet Child E' Adolescent Health, 4, 397-404. https://doi.org/10.1016/S2352-4642(20)30095-X

Williamson, B. (2018). Big data en educación. El futuro digital del aprendizaje, la política y la práctica. Morata.

\section{Breve CV del autor}

\section{Guillermo Ramón Ruiz}

Doctor en Ciencias de la Educación, Universidad de Buenos Aires (UBA), y Master of Arts in Education, University of California Los Angeles (UCLA). Es Profesor Titular Regular (Catedrático) en las Facultades de Derecho y de Psicología de la UBA. Es Investigador del Consejo Nacional de Investigaciones Científicas y Técnicas (CONICET). Sus actividades de investigación refieren a las reformas educativas de la educación secundaria, al debate pedagógico sobre el contenido material del derecho a la educación, y a las políticas de formación de profesorado en perspectiva internacional y comparada. Es autor de libros $\mathrm{y}$ artículos científicos de $\mathrm{su}$ especialidad. ORCID ID: https://orcid.org/OOOO-OO01-8170-2191. Email: gruiz@derecho.uba.ar 